\title{
Nonequilibrium Dynamics of Anisotropic Large Spins in the Kondo Regime: Time-Dependent Numerical Renormalization Group Analysis
}

\author{
David Roosen, ${ }^{1}$ Maarten R. Wegewijs, ${ }^{2,3}$ and Walter Hofstetter ${ }^{1}$ \\ ${ }^{1}$ Institut für Theoretische Physik, Johann Wolfgang Goethe-Universität, 60438 Frankfurt/Main, Germany \\ ${ }^{2}$ Institut für Festkörper-Forschung-Theorie 3, Forschungszentrum Jülich, 52425 Jülich, Germany \\ ${ }^{3}$ Institut für Theoretische Physik A, RWTH Aachen, 52056 Aachen, Germany
}

(Received 25 May 2007; published 27 February 2008)

\begin{abstract}
We investigate the time-dependent Kondo effect in a single-molecule magnet (SMM) strongly coupled to metallic electrodes. Describing the SMM by a Kondo model with large spin $S>1 / 2$, we analyze the underscreening of the local moment and the effect of anisotropy terms on the relaxation dynamics of the magnetization. Underscreening by single-channel Kondo processes leads to a logarithmically slow relaxation, while finite uniaxial anisotropy causes a saturation of the SMM's magnetization. Additional transverse anisotropy terms induce quantum spin tunneling and a pseudospin-1/2 Kondo effect sensitive to the spin parity.
\end{abstract}

DOI: 10.1103/PhysRevLett.100.087201

Introduction.-Crystals of single-molecule magnets (SMMs) are mesoscopic systems, which exhibit fundamental quantum mechanical phenomena like quantum tunneling of the magnetization (QTM). The individual SMMs responsible for these effects have large spin $S \sim 10$ and show rich quantum dynamics due to the anisotropic effective potential induced by ligand-fields and spin-orbit coupling [1]. More recently, transport through individual SMMs coupled to metallic electrodes has been measured for the first time [2,3], giving access to many-body physics in these complex quantum impurity systems. In particular, the large spin, in combination with magnetic anisotropy, was predicted to give rise to a pseudospin-1/2 Kondo effect [4] in a single SMM. In combination with applied magnetic fields, this effect can be used for transport spectroscopy of an individual SMM in a two-terminal geometry [5]. Beyond the linear response transport investigated in these works, one can gain fundamental insight by studying nonequilibrium magnetization dynamics of an SMM coupled to macroscopic electrodes. Recent STM experiments have also demonstrated that single molecules attached to a substrate can be abruptly switched to a new conformation with different transport properties by a voltage pulse, resulting, for instance, in a change in the Kondo temperature [6]. Recent advances in the field of quantum computing using electron spins in semiconductor quantum dots concern high-frequency control of magnetic and electric signals. In particular, recently it has been shown experimentally [7] that via oscillating electric fields spin transitions can be induced locally by spin-orbit coupling. There are prospects for applying such techniques to threeterminal single-molecule devices although technical challenges remain.

Motivated by these advances, here we theoretically analyze the nonequilibrium spin dynamics of a SMM in response to a sudden change of the magnetic field strength, with a special focus on the manifestation of Kondo screening in the time domain. The accurate real-time calculation
PACS numbers: 75.50.Xx, 31.70.Hq, 72.15.Qm, 75.45.+j

of impurity observables like the magnetization is crucial for developing a general understanding of time-resolved Kondo spectroscopy of SMMs, for instance, using transport measurements [5]. More generally, proposals for spintronic operation of SMM-based devices [8-12] rely on a detailed understanding of the magnetization dynamics. Note that here we consider pulsed magnetic fields as opposed to the experimentally more easily accessible oscillating fields, since the former are at the moment more conveniently treated using numerical renormalization group (NRG) methods [13-16]. In order to analyze the effects of pulsed fields we use the time-dependent numerical renormalization group method (TD-NRG) recently introduced by Anders and Schiller [16]. The advantage of this approach is that the time evolution of any quantum impurity system after a sudden perturbation at $t=0$ can be calculated in a numerically exact way on arbitrarily long time scales without accumulating any discretization error that scales with the elapsed time, in contrast, e.g., to the adaptive time-dependent DMRG [17]. For a detailed description of the TD-NRG method we refer to [16]. For all the results presented below we have exhaustively checked that the dependence on the method's parameters is negligible (such as the discretization parameter $\Lambda$ or the number of states $N_{\text {Level }}$ taken into account in each iteration). We employ units in which $g=\hbar=k_{B}=W=1$, where $W$ is half the width of the conduction band.

Model. - We consider a SMM as a (generalized) quantum impurity coupled to a conduction band of metallic electrodes: $\mathcal{H}=\mathcal{H}_{\mathrm{cb}}+\mathcal{H}_{\text {int }}+\mathcal{H}_{\text {imp }}$ [4]. The conduction band and the interaction term in the Hamiltonian read $\mathcal{H}_{\mathrm{cb}}=\sum_{k, \mu} \varepsilon_{k} c_{k \mu}^{\dagger} c_{k \mu}, \mathcal{H}_{\mathrm{int}}=J \mathbf{S} \cdot \mathbf{s}$, where $\mathbf{s}$ is the spin of the most localized electronic orbital in the conduction band which is coupled to the impurity spin $\mathbf{S}$ (e.g., $S=10$ for the single-molecule magnet $\mathrm{Mn}_{12}$ and $S=8$ in case of $\mathrm{Fe}_{8}$ ). Below we systematically investigate different models describing the impurity $\mathcal{H}_{\text {imp }}$ (see Fig. 1). The time scale 


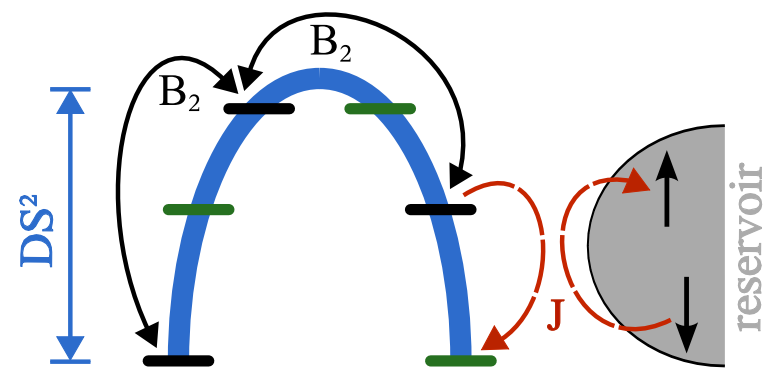

FIG. 1 (color online). Energy diagram of the SMM. The levels indicate spin eigenstates $|S, M\rangle$ which are split in energy by the uniaxial $(D)$ anisotropy. Two types of spin fluctuations occur: processes due to the intrinsic transverse anisotropy $\left(B_{2}\right)$, resulting in QTM, and exchange processes involving the conductionband electrons $(J)$, leading to the Kondo effect.

on which physical quantities vary throughout this work typically ranges from $10^{3}$ to $10^{9}$ times $\hbar / W$ corresponding to $1 \mathrm{ps}-1 \mu \mathrm{s}$ for typical values of the spin, exchange interaction, and anisotropy parameters.

Isotropic Kondo model. - Let us first investigate an isotropic spin $S$ with antiferromagnetic exchange $J>0$ induced by electron tunneling, i.e., $\mathcal{H}_{\text {imp }}=0$. For a single electron with spin $S=1 / 2$ at zero temperature and zero magnetic field the local moment is completely screened by the Kondo effect, i.e., $\left\langle S_{z}\right\rangle=0$. Here, however, we consider a large impurity spin $S>1 / 2$, as relevant for SMMs [4], aligned at times $t<0$ by a strong magnetic field $h_{z}$ in the longitudinal direction, which is sufficiently strong to completely polarize the impurity spin. At $t=0$ the field is switched off and using the TD-NRG we calculate the screening of the SMM's magnetization by exchange scattering processes as a function of time, see Fig. 2. The most prominent observation is that, in contrast to the $S=1 / 2$ case, the interaction between the impurity and the conduction electrons does not quench the magnetic moment on the

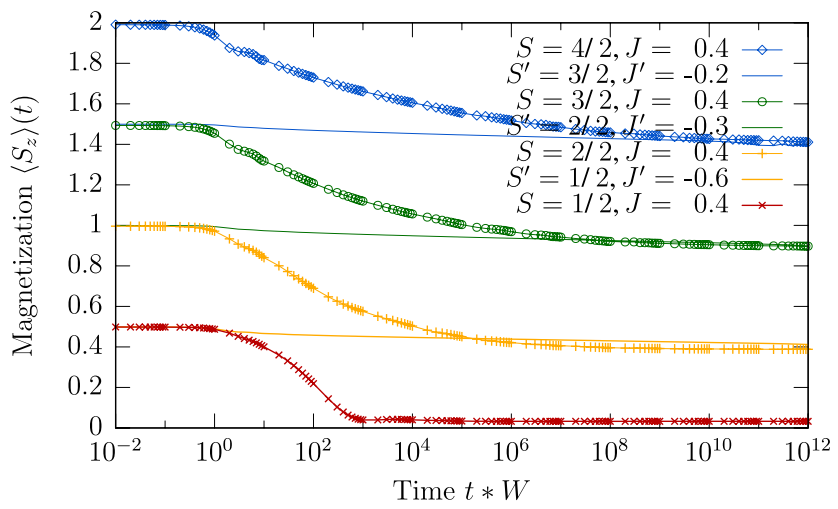

FIG. 2 (color online). The logarithmic decay of $\left\langle S_{z}\right\rangle(t)$ due to underscreening of the local moment for different spin sizes $S$ $(J=0.4)$ is compared to the relaxation of a spin $S^{\prime}=S-1 / 2$, whose effective ferromagnetic coupling $J^{\prime}<0$ is chosen according to [22]. (NRG parameters: $\Lambda=2.5, N=60, N_{\text {Level }}=$ 2000). impurity completely. This is referred to as the underscreened Kondo effect [18-22]. In a naïve picture the equilibrium underscreened Kondo effect can be described as a two-stage process: first the magnetization is reduced to $S-1 / 2$ by screening due to the spin- $1 / 2$ electrons in the conduction band. This effective spin $S^{\prime}=S-1 / 2$ then couples ferromagnetically $\left(J^{\prime}<0\right)$ to the conduction band and becomes asymptotically free at low energies [19]. Our results in Fig. 2 show how this energy dependence of the effective couplings translates into the real-time dynamics of the impurity spin. For different values of $S$ and $J=0.4$ we observe an initial drop of the magnetization by approximately $1 / 2$, followed by a logarithmically slow further relaxation (note that $\left\langle S_{z}\right\rangle$ is not a conserved quantity and can drop below the value of $S_{z}-1 / 2$ ). Comparing the low energy excitations for a spin $S$ and antiferromagnetic coupling to those of a spin $S^{\prime}=S-1 / 2$ impurity coupled ferromagnetically, Koller et al. [22] have determined the value of the renormalized ferromagnetic interaction $J^{\prime}$ for the underscreened Kondo effect. In Fig. 2 we show that this correspondence can also be observed in the real-time dynamics, by comparing the underscreened $J=0.4$ relaxation dynamics with a ferromagnetic $S^{\prime}=S-1 / 2$ Kondo impurity and an effective $J^{\prime}<0$ as calculated in [22]. In the ferromagnetic case, we fitted the time evolution as $\left\langle S_{z}(t)\right\rangle=A /[\log (t+B)]$ using $A$ and $B$ as fit parameters and found very good agreement with our results. For antiferromagnetic coupling good asymptotic agreement with the corresponding ferromagnetic case was found in the time evolution. This confirms our identification of timeresolved underscreening. We observe that the time scale of the partial screening $S \rightarrow S-1 / 2$ increases with the spin size $S$. If the exchange interaction $J$ is decreased, the effect is more dramatic: the partial screening sets in at larger times and its time scale increases considerably (not shown) in agreement with the nonlinear increase of the effective coupling $J^{\prime}$ for small $J$ as found in [22].

Kondo model with uniaxial anisotropy. - Singlemolecule magnets are characterized by additional magnetic anisotropy terms in the impurity part of the Hamiltonian, as described by the generic model $\mathcal{H}_{\text {imp }}=$ $-D S_{z}^{2}-\frac{1}{2} B_{2}\left(S_{+}^{2}+S_{-}^{2}\right)-\mathbf{h} \cdot \mathbf{S}$. The spin has to overcome an energy barrier of height $D S_{z}^{2}$ to reverse its ground state alignment with the easy axis intrinsic to the SMM. Ligand field effects reduce the symmetry group of the molecule about this axis down to a finite group. The noncommuting terms $\propto B_{2}$ allowed by this low symmetry introduce quantum tunneling of the magnetization (QTM) through the barrier [1]. The energy levels of an isolated SMM are sketched in Fig. 1. The resulting model has recently been studied [4,5] since it is relevant to transport experiments on SMMs immobilized between metallic electrodes [2,3].

We first investigate how the development of the underscreened Kondo effect is hampered by a finite anisotropy barrier $D>0$ in the absence of QTM $\left(B_{2}=0\right)$. When 
switching off the magnetic field, a clear saturation of the magnetization on a time scale $\propto 1 / D$ is seen in Fig. 3. The logarithmic decay characteristic of the underscreened Kondo effect without anisotropy $D$ is terminated, because Kondo spin fluctuations with small energy uncertainty are suppressed by the barrier. In a renormalization group language, the scaling is cut off at $D$ and the Kondo screening stays incomplete.

Kondo model with uniaxial and transverse anisotropy.The transverse anisotropy term $B_{2}$ induces QTM in SMMs in the absence of a magnetic field. We first consider the typical case $B_{2} \ll D$ and strong coupling to the conduction band $J=0.2$ for half-integer spins $S>1 / 2$. The Kondo effect developing in this limit was shown [4] to involve all magnetic excitations of the SMM, since $T_{K}\left(S, J, B_{2}, D\right)$ exceeds the magnetic splittings due to the large $J$ value. As before, we prepare a spin $S=3 / 2$ system in an almost completely polarized state $\left\langle S_{z}\right\rangle(0) \approx 3 / 2$ at $t<0$, and monitor the subsequent time dependence of the magnetization $\left\langle S_{z}\right\rangle(t)$. For very weak transverse anisotropy $(D=$ $\left.5 \times 10^{-7}, B_{2}=8 \times 10^{-9}, J=0.2\right)$, three different regimes can be distinguished in the time evolution (cf. Fig. 4): (i) starting at $t \approx 1 / W$ the conduction-band electrons partially screen the spin on the impurity. Both the time evolution and the NRG energy levels are the same as for the "bare" underscreened Kondo effect obtained by setting $B_{2}=D=0$. (ii) The transverse anisotropy $B_{2}$ introduces a new feature: for times $t W>100$ (i.e., before the finite barrier $D$ "freezes" the spin) the magnetization starts declining and displays an oscillation around the value $\left\langle S_{z}\right\rangle \approx 0.35$. (iii) The remaining magnetization is quenched by spin exchange processes between the conduction-band electrons and the impurity. The time scale is given by the inverse Kondo temperature of the pseudospin-1/2 Kondo effect $\tau \propto 1 / T_{K}\left(S, J, B_{2}, D\right)$ in line with $[4,5]$. The small remnant magnetization at large times is due to incomplete thermalization which is unavoidable in a finite size system with discretization parameter $\Lambda>1$; see also [16].

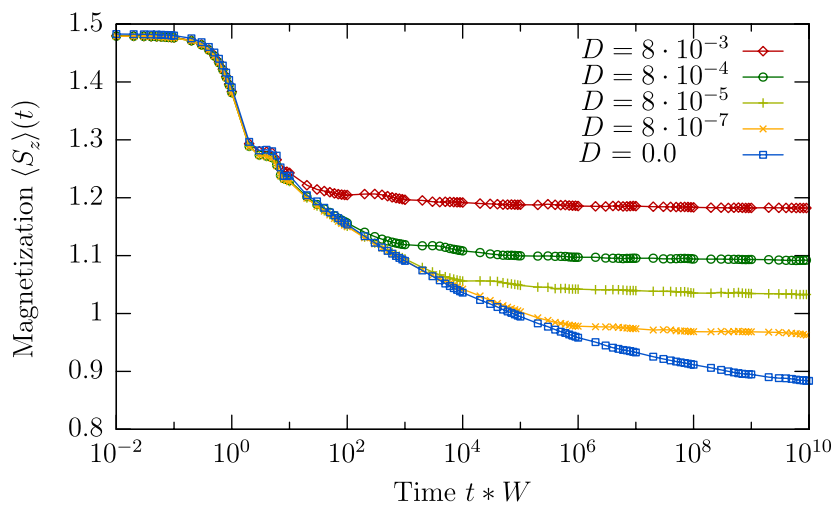

FIG. 3 (color online). Suppression of underscreening: the magnetic anisotropy barrier $D>0$ impedes further relaxation of the SMM's magnetization by the Kondo effect $(S=3 / 2, J=0.6)$. (NRG parameters: $\Lambda=1.8, N=60, N_{\text {Level }}=1000$ ).
In order to study the sensitivity of the observed magnetization dynamics to the spin size $S$ as well as the exchange coupling $J$, we now consider large values for the anisotropy barrier $D \sim 10^{-2} W$ and the quantum tunneling term $B_{2} \sim$ $D$, for a weakly coupled SMM $(J=0.1 W)$. For halfinteger spin $S$ the quantum tunneling should then reduce the magnetization to a finite value, before the underscreened Kondo effect sets in. Our results for the timedependent magnetization $\left\langle S_{z}\right\rangle(t)$ indeed display this behavior: Fig. 5(a) shows a clear two-step relaxation and a strong dependence on the half-integer value of $S$ as well as on the exchange coupling $J$. At $t W \approx 10$ the quantum tunneling term $B_{2}$ starts mixing the different magnetic states of the molecule. The magnetization is reduced to $\left\langle S_{z}\right\rangle \approx 0.7$ showing damped Rabi-type oscillations around this value for all spin sizes we investigated $(S=3 / 2-7 / 2)$, which are related to the quantum tunneling term and which disappear for $B_{2}=0$. For larger times $t$, we again find the pseudospin-1/2 Kondo effect to set the relevant time scale $\tau \propto 1 / T_{K}\left(S, J, B_{2}, D\right)$ for the complete screening of the molecule's magnetization. Since the Kondo temperature increases with the size of the spin (cf. [4]) the relaxation is faster for larger spin values $S$ [cf. Fig. 5(a)]. The dependence of the relaxation on the exchange coupling $J$ for fixed spin $S=5 / 2$ is shown in the inset of Fig. 5(a). For

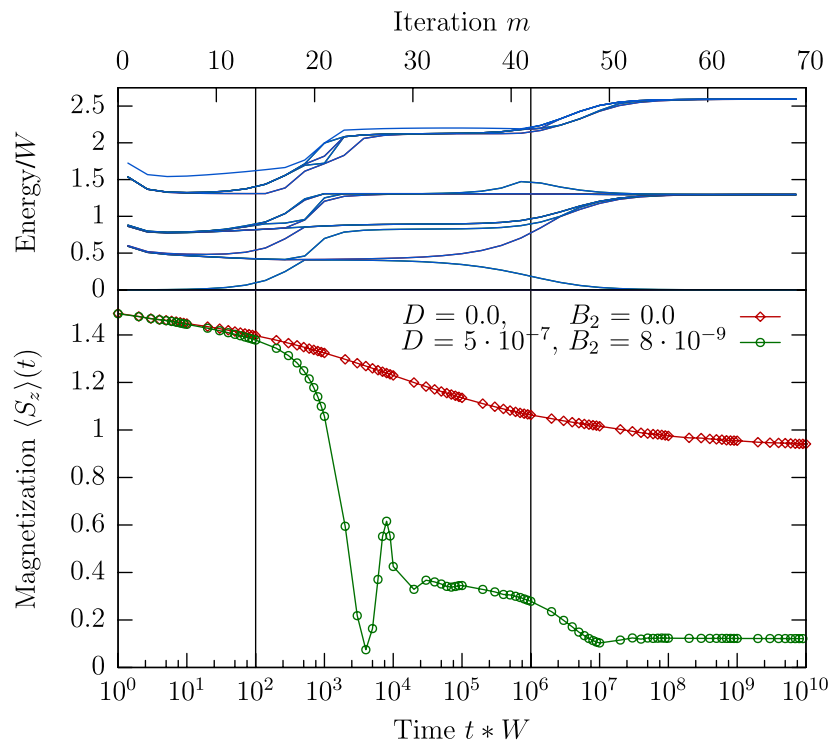

FIG. 4 (color online). Half-integer spin $(J=0.2)$. Three different regimes in the time evolution relate to underscreening $(t W<100)$, buildup of the pseudospin-1/2 Kondo effect $(100<$ $\left.t W<10^{6}\right)$, and approach to the strong-coupling fixed point $\left(t W>10^{6}\right)$. Above the time evolution we plot the eigenenergies of the NRG Hamiltonian $H_{m}$ as a function of iteration $m$, i.e., the spectrum of the many-body system SMM + electrode, where conduction-band electrons with energies above $1 / \Lambda^{m}$ have been integrated out exactly. The scales for the two plots are adjusted to each other, allowing a direct comparison between the NRG level flow and the corresponding real-time evolution. (NRG parameters: $\Lambda=2.0, N=70$.) 

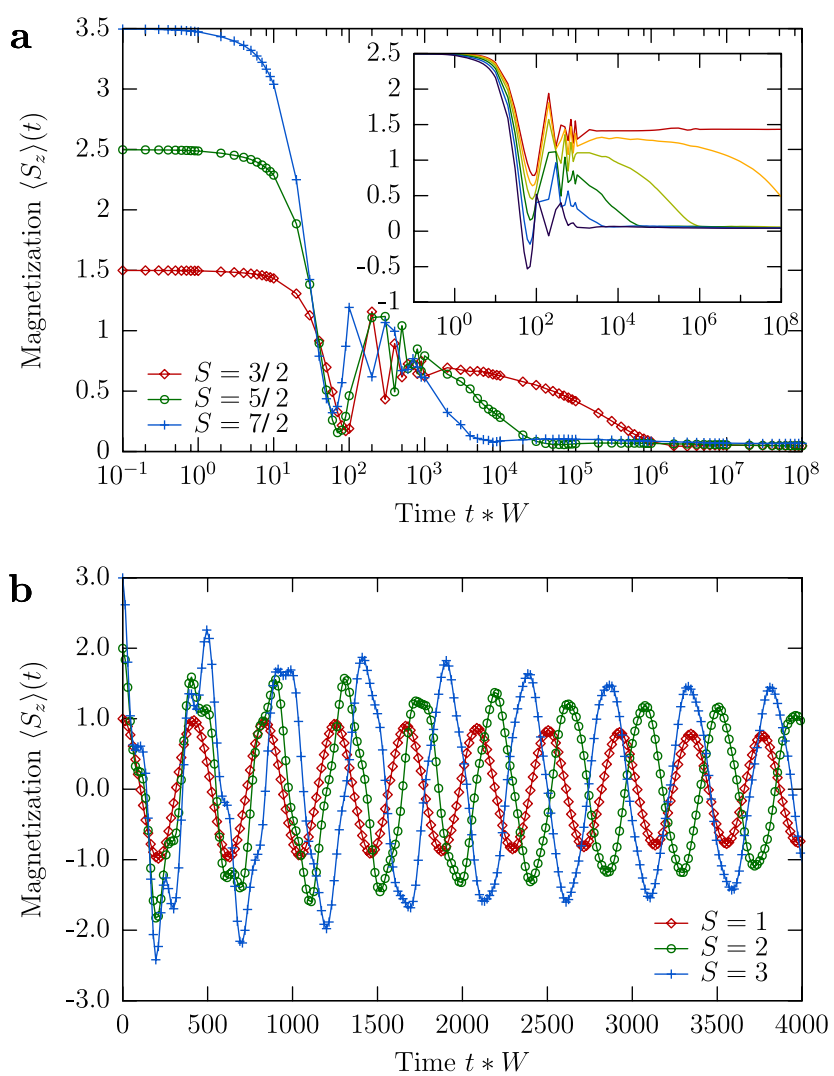

FIG. 5 (color online). Magnetization dynamics for weak Kondo coupling $J=0.1$, large QTM, $B_{2}=0.75 \times D=7.5 \times$ $10^{-3}$, and different sizes of the spin. (a) Half-integer spin: twostage relaxation, with a time scale for the Kondo effect strongly dependent on the size of the spin. Inset: dependence on exchange coupling $J$ [shown for spin $S=5 / 2, J=0.001$ (red) and $J=$ 0.05 (orange) ...0.15 (purple) in equidistant steps]. (b) Integer spin: the different spin sizes have only little effect on the damped Rabi oscillation of the magnetization around zero. (NRG parameters: $\Lambda=2.0, N=65$.)

larger $J$ the oscillations reach negative values. The narrow plateau after the first "drop" in the magnetization, which is due to the transverse anisotropy $B_{2}$, saturates at $\left\langle S_{z}\right\rangle \approx 1.5$, and the time scale for the pseudospin- $1 / 2$ Kondo effect becomes very large in the limit of weak coupling $J$.

For integer spin values $S$ the QTM term alone allows the magnetization to tunnel between the two ground states $|S\rangle$ and $|-S\rangle$ of the isolated SMM $(J=0)$, resulting in zero net equilibrium magnetization at low magnetic field. Therefore, one does not expect a Kondo effect, because no cooperation of the spin exchange processes is needed to change the direction of the magnetization [4]. We indeed observe a dramatic change in the spin dynamics going from half-integer spin to the nearest full-integer $S$ for identical parameters $B_{2}, D$, and $J$ [compare Fig. 5(a) and 5(b)]. $\left\langle S_{z}\right\rangle(t)$ displays an oscillation around zero which dies out on time scales $\sim 10^{4} / W$ due to damping induced by the conduction-band electrons.
Conclusions. - In this work we have analyzed the nonequilibrium magnetization dynamics of Kondo models with large spin $S>1 / 2$ in response to a sudden perturbation. For the isotropic Kondo model we found a two-stage relaxation, which displays the underscreened Kondo effect in the real-time magnetization dynamics and confirms that the renormalized coupling to the impurity after a partial screening of the magnetization to $S-1 / 2$ is ferromagnetic $J^{\prime}<0$. For the extended anisotropic Kondo Hamiltonian describing a single-molecule magnet, we found a suppression of the underscreened Kondo effect by uniaxial anisotropy. With additional transverse anisotropy, relaxation becomes sensitive to the spin parity. For half-integer spin, a complex interplay of tunneling and spin screening by conduction electrons leads to a pseudospin-1/2 Kondo effect and a reduction of the magnetization on long time scales. In contrast, for integer spin, the transverse anisotropy only leads to damped Rabi oscillations without spin screening.

The authors thank Christian Romeike for discussions.

[1] D. Gatteschi and R. Sessoli, Angew. Chem., Int. Ed. Engl. 42, 268 (2003), and references therein; W. Wernsdorfer and R. Sessoli, Science 284, 133 (1999).

[2] H. Heersche et al., Phys. Rev. Lett. 96, 206801 (2006).

[3] M.-H. Jo et al., Nano Lett. 6, 2014 (2006).

[4] C. Romeike et al., Phys. Rev. Lett. 96, 196601 (2006).

[5] C. Romeike et al., Phys. Rev. Lett. 97, 206601 (2006).

[6] V. Iancu, A. Deshpande, and S.-W. Hla, Nano Lett. 6, 820 (2006).

[7] K. C. Nowack et al., Science 318, 1430 (2007), and references therein.

[8] C. Romeike, M. R. Wegewijs, and H. Schoeller, Phys. Rev. Lett. 96, 196805 (2006).

[9] C. Timm and F. Elste, Phys. Rev. B 73, 235304 (2006).

[10] M. Misiorny and J. Barnas, Europhys. Lett. 78, 27003 (2007).

[11] G. Gonzalez and M. N. Leuenberger, Phys. Rev. Lett. 98, 256804 (2007).

[12] J. Lehmann and D. Loss, Phys. Rev. Lett. 98, 117203 (2007).

[13] K. G. Wilson, Rev. Mod. Phys. 47, 773 (1975).

[14] W. Hofstetter, Phys. Rev. Lett. 85, 1508 (2000).

[15] T. A. Costi, Phys. Rev. B 55, 3003 (1997).

[16] F. Anders and A. Schiller, Phys. Rev. Lett. 95, 196801 (2005); Phys. Rev. B 74, 245113 (2006).

[17] A. J. Daley et al., J. Stat. Mech. (2004) P04005; S. R. White and A. E. Feiguin, Phys. Rev. Lett. 93, 076401 (2004).

[18] P. Nozieres and A. Blandin, J. Phys. (Paris) 41, 193 (1980).

[19] D. M. Cragg and P. Lloyd, J. Phys. C 12, L215 (1979).

[20] A. M. Tsvelick and P. B. Wiegmann, Adv. Phys. 32, 453 (1983); N. Andrei, K. Furuya, and J. H. Lowenstein, Rev. Mod. Phys. 55, 331 (1983).

[21] P. Coleman and C. Pepin, Phys. Rev. B 68, 220405 (2003).

[22] W. Koller, A. C. Hewson, and D. Meyer, Phys. Rev. B 72, 045117 (2005). 